\title{
PERFIL DAS PUBLICAÇÕES CIENTÍFICAS BRASILEIRAS SOBRE A PSICOLOGIA SOCIAL PICHONIANA
}

\author{
Lucas Magalhães Cruz*, Verena Dalton Teles Jesus Barbosa** \\ Autor correspondente: Lucas Magalhães Cruz - lucasmcpsi@gmail.com \\ * Psicólogo formado pela Universidade Salvador (UNIFACS). Discente do Curso de Especialização em Psicologia Social do \\ Centro Interdisciplinar de Estudos Grupais Enrique Pichon-Rivière (CIEG) \\ ** Psicóloga formada pela Universidade Salvador (UNIFACS). Gestalt-terapeuta pelo Instituto de Gestalt-terapia da Bahia \\ (IGTBa)
}

\section{Resumo}

Este artigo é resultado de uma pesquisa bibliográfica exploratória que teve como objetivo traçar o perfil da produção científica brasileira referente à psicologia social de Enrique Pichon-Rivière nas bases de dados do Scielo (Scientific Electronic Library Online) e do Pepsic (Periódicos Eletrônicos de Psicologia) com os descritores "pichon" e "grupo operativo". Foram encontrados 90 artigos, sendo 56 compatíveis com os critérios de inclusão na pesquisa, os quais foram analisados com base nas seguintes classificações: título do trabalho, ano de publicação, periódico de publicação, objeto de estudo e/ou intervenção, tipo de estudo, temáticas dos artigos, formação do(s) autor(es), sexo do(s) autor(es) e número de autor(es). O tipo de formação dos autores também foi analisado com base nos dados contidos na plataforma Lattes sobre eles. Por fim, reflexões e discussões sobre as informações encontradas compõem um panorama das publicações sobre psicologia social pichoniana no Brasil. Palavras-chave: Pichon-rivière; Psicologia social; Pesquisa bibliográfica; Grupo operativo.

\section{SCENE OF THE PICHONIAN SOCIAL PSYCHOLOGY IN BRAZILIAN SCIENTIFIC PUBLICATIONS}

\begin{abstract}
This article is the result of exploratory bibliographic research which sought to trace the brazilian scientific production scene related to the social psychology of Enrique Pichon-Rivière published in Scielo (Scientific Electronic Library Online) and Pepsic (Periódicos Eletrônicos de Psicologia) databases under "pichon" and "grupo operativo" descriptors. There, among 90 manuscripts found, 56 were compatible with the inclusion criteria of the research under the following classification: title, publishing year, publication type, object of study or intervention, study type, articles' thematic essay, authors' degrees, gender and
\end{abstract}


quantity of authors per work. The authors' credentials were assessed under the information available on the Lattes platform. Summing up, reflections and discussions about the findings contrive a scene of the pichonian social psychology publications in Brazil.

Keywords: Pichon-rivière; Social psychology; Bibliographic research; Operative group.

\section{INTRODUÇÃOO}

O presente trabalho tem como objetivos conhecer o estado da publicação de artigos científicos que utilizam como referência a psicologia social pichoniana no Brasil e delinear o perfil destas produções. Esta proposta se configura como relevante devido à valorização da teoria de Enrique Pichon-Rivière e da sua técnica dos grupos operativos nas mais variadas instituições.

Médico psiquiatra, Pichon-Rivière fundou a primeira escola privada de psicologia social em 1967, época de muitos questionamentos à psicologia social acadêmica e fortalecimento de perspectivas revolucionárias na Argentina. ${ }^{(1)}$ A proposta de Pichon-Rivière fazia uma crítica a esta psicologia social acadêmica e tinha uma perspectiva transformadora.

As ideias de Pichon-Rivière foram ganhando espaço, tendo, ao longo dos anos, os seus conteúdos incorporados em alguns cursos de ensino superior. Atualmente as instituições de formação fora da universidade se mantêm e são reconhecidas quanto ao ensino da psicologia social pichoniana. ${ }^{(1)} \mathrm{No}$ Brasil, a teoria de Pichon-Rivière também é ensinada em universidades e em instituições formadoras.

$\mathrm{Na}$ área da saúde pública, há um incentivo ao trabalho com grupos, e isso é perceptível, por exemplo, na Política Nacional de Humanização da Saúde que propõe a criação de espaços de troca coletiva com a criação de grupos. ${ }^{(2,3)} \mathrm{E}$ também podem ser vistos que, em algumas publicações oficiais do Ministério da Saúde, há referências específicas à técnica de grupos operativos.
Em um manual sobre saúde mental, ao citar as atividades dos Centros de Atenção Psicossocial (CAPS), encontra-se: "4.1.1 - A assistência prestada ao paciente no CAPS I inclui as seguintes atividades: [...] b - atendimento em grupos (psicoterapia, grupo operativo, atividades de suporte social, entre outras)".(4)

$\mathrm{Na}$ portaria que institui unidades de acolhimento para pessoas que usam álcool e outras drogas consta o seguinte:

\footnotetext{
Art. 10. As ações a serem desenvolvidas pelas Unidades de Acolhimento e o tempo de permanência de cada usuário deverão estar previstas no Projeto Terapêutico Singular [...] VI - atendimento em grupos, tais como psicoterapia, grupo operativo, atividades de suporte social, assembleias, grupos de redução de danos, entre outros. ${ }^{(5)}$
}

A técnica de grupo operativo, citada como um dispositivo a ser utilizado na saúde, é centrada em uma tarefa grupal, na elaboração das ansiedades e com a finalidade "que seus integrantes aprendam a pensar em uma coparticipação do objeto de conhecimento, entendendo-se que pensamento e conhecimento não são fatos individuais, mas produções sociais". ${ }^{(6)}$ Apesar do destaque nas publicações da área de saúde pública, deve-se pontuar que esta técnica não pode ser dissociada da teoria da psicologia social pichoniana. ${ }^{(7)}$

Esta técnica tem uma série de especificidades e características que a diferem de outras formas de abordagem em grupo. Há, portanto, uma comple- 
xidade em relação tanto à técnica quanto à bagagem teórica interdisciplinar de Pichon-Rivière, o que torna fundamental uma formação para poder utilizar a técnica dos grupos operativos.

A teoria desenvolvida por Pichon-Rivière se caracteriza por ter um Esquema Conceitual Referencial Operativo (ECRO) que significa um conjunto interdisciplinar de teorias, noções e conceitos que dizem respeito a um setor do real.(6) É este ECRO que organiza o arcabouço teórico da psicologia social pichoniana. Como escreveu Pichon-Rivière, "estas contribuições são provenientes do materialismo dialético, do materialismo histórico, da psicanálise, da semiologia e das contribuições daqueles que trabalham em uma interpretação totalizadora das relações entre estrutura socioeconômica e vida psíquica". ${ }^{(6)}$

Considerando sua complexidade teórica e as demandas da utilização da técnica no cenário nacional, faz-se pertinente a realização de pesquisas que procurem compreender como está a produção de conhecimento dentro dessa abordagem. Neste sentido, podem-se ver quais caminhos estão sendo traçados e quais trajetórias demandam mais estudos, de acordo com as possibilidades que a teoria pichoniana oferece, de modo a balizar as práticas profissionais e outras pesquisas sobre a temática.

\section{MÉTODO}

Esta pesquisa se caracteriza como do tipo exploratória, que como Gil define, "tem como objetivo proporcionar maior familiaridade com o problema, com vistas a torná-lo mais explícito ou a constituir hipóteses". ${ }^{(8)}$ Dentro desta proposta de explorar a atual situação de publicações, se delineou uma pesquisa bibliográfica exclusivamente com artigos científicos brasileiros em acesso aberto.

Os artigos foram coletados nos meses de outubro e novembro de 2014, no banco de dados do Scientific Electronic Library Online (Scielo) que é uma biblioteca eletrônica que contempla uma série de periódicos científicos e do Periódicos Eletrônicos de Psicologia (Pepsic), um portal de periódi- cos eletrônicos de Psicologia com uma importante função na divulgação do conhecimento psicológico latino-americano. Foram utilizados os descritores "pichon" e "grupo operativo", e os critérios de inclusão foram que os artigos estivessem integralmente em português e contendo referências à psicologia social de Enrique Pichon-Rivière.

Após a seleção dos artigos pesquisados, foi realizada uma classificação com base nas seguintes dimensões: título do trabalho; ano de publicação; periódico de publicação; objeto de estudo e/ou intervenção; tipo de estudo; temáticas dos artigos; formação do(s) autor(es); sexo do(s) autor(es); número de autor(es). Sendo que, a partir dos resultados, se criaram novas classificações, por exemplo: nos estudos sobre uma intervenção, se delineou onde ocorreu a intervenção, qual a natureza do lugar e seu público-alvo.

Em relação à formação dos autores, foram observados os cursos relacionados à psicologia social pichoniana, além dos títulos dos trabalhos finais de dissertações de mestrados e teses de doutorados em que constavam os descritores "pichon" e "grupos operativos". Para isto, foram pesquisados os tópicos "formação acadêmica/titulação" e "formação complementar", nos currículos dos autores e coautores disponíveis na Plataforma Lattes.

\section{RESULTADOS}

No total, nas duas bibliotecas eletrônicas, foram encontrados 90 artigos. Como alguns não se referiam propriamente à psicologia social pichoniana $\mathrm{e}$ outros estavam duplicados por pertencer a revistas científicas hospedadas nas duas bibliotecas, foram enfim selecionados 56 artigos.

Em relação ao perfil dos autores, os 56 artigos coletados foram escritos por 135 autores, $77,04 \%$ do sexo feminino e $22,96 \%$ do sexo masculino. Do total de artigos, em apenas $41,07 \%$ deles havia algum autor com formação relacionada aos descritores "pichon" e "grupos operativos" no seu currículo Lattes. Destes, 16 indicavam ter formação ou pós-graduação lato sensu na área, em cinco cur- 
rículos constava mestrado relacionado, em dois, doutorado, e nove nomeavam curso(s) com carga horária menor do que 100 horas.

Os cursos de graduação dos autores que possuíam esse dado no seu currículo Lattes foram: 37 formados em psicologia (54\%), 20 em enfermagem (29\%), três em medicina (4\%), três em educação $(4 \%)$, dois em educação física (3\%), um em fisioterapia, um em nutrição, um em administração ( $2 \%$ cada). O gráfico 1 representa o predomínio de graduações ligadas à área de saúde.

Gráfico 1 - Formação dos autores

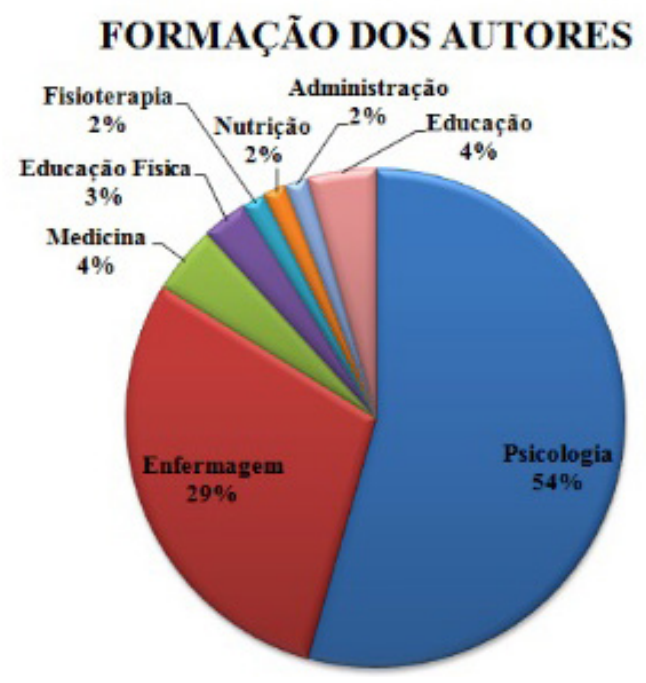

Percebeu-se que os autores de diferentes formações escreveram artigos juntos, tendo as seguintes conformações: enfermagem e medicina; psicologia, educação física e medicina; fisioterapia e psicologia; enfermagem e psicologia; nutrição e psicologia; enfermagem, psicologia e medicina; administração e psicologia; e educação e psicologia.
Em relação aos anos de publicação, notou-se um grande aumento de publicações no século XXI, pois até 2001 foram publicados apenas quatro artigos, para um crescimento de no mínimo três publicações em cada ano, como se pode ver no gráfico 2 .

Gráfico 2 - Publicação por ano

\section{PUBLICAÇÃO POR ANO}

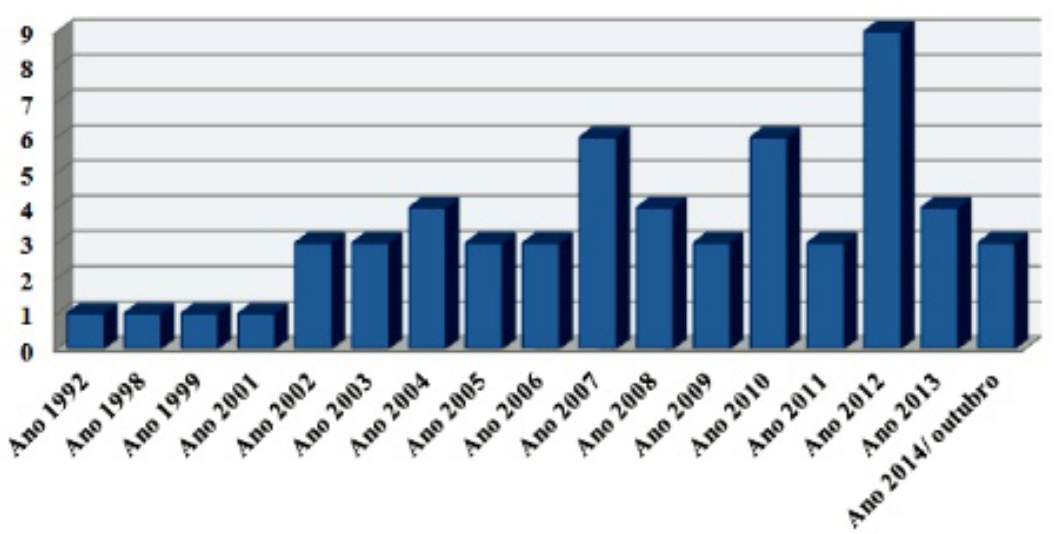


Os artigos foram publicados em diversas as revistas científicas, no total de 31 , sendo $51,61 \%$ revistas de psicologia e $22,58 \%$ de enfermagem. As outras também eram da área de saúde de maneira geral ou educação.

Para classificar os artigos segundo a abrangência da utilização da teoria pichoniana e/ou a técnica de grupos operativos, na dimensão do tipo de estudo, resolveu-se utilizar a nomenclatura "intervenção com grupos operativos", "intervenção com conceitos pichonianos", "ensaio teórico" e "outros". A primeira categoria se refere a artigos que fazem utilização da técnica dos grupos operativos em um contexto específico; a segunda trata de artigos que não relatam o uso da técnica dos grupos operativos, mas que utiliza os conceitos de Pichon-Rivière para teorizar uma intervenção; "ensaio teórico" é um artigo que usa os conceitos pichonianos, mas não relata uma intervenção. Na categoria "outros", foram incluídos artigos que, por exemplo, traziam o relato de entrevistados que citavam a teoria de Pichon-Rivière, mas esta teoria não era objeto de estudo nem fazia parte da discussão teórica.

Foram $37,5 \%$ de estudos do tipo "intervenção com grupos operativos", $28,6 \%$ do tipo "intervenção com conceitos pichonianos", $23,2 \%$ do tipo "ensaio teórico" e 10,7\% incluídos entre "outros". Houve, portanto, uma predominância de artigos com intervenções, com ou sem utilizar a técnica dos grupos operativos, num percentual de $66,1 \%$.

Os ensaios teóricos tiveram como assunto principal a explicação de conceitos pichonianos como a tarefa grupal e também apresentavam uma introdução à teoria de Pichon-Rivière. Outros tinham a proposta de articular os conceitos de Pichon-Rivière com os de outros teóricos, como Henri Wallon ou Paulo Freire. Alguns artigos apenas apresentavam uma das ideias de Pichon-Rivière frente a outros teóricos para fundamentar uma discussão. $E$ um dos artigos realizou uma análise de um filme a partir da ótica pichoniana.

Quanto aos campos dos artigos interventivos, as intervenções ocorreram em: "saúde pública" (47,8\%), "saúde mental" (4,5\%), "formação de ensino superior" $(22,7 \%)$, "escolas e projetos profissionalizantes" (11,4\%), "projetos sociais e comunidades" ( $9,1 \%)$, "movimentos sociais" (2,3\%), e outros $(2,3 \%)$. Vale esclarecer que o campo de saúde mental foi colocado à parte da saúde pública para realçar essa área específica onde a técnica dos grupos operativos é mais citada. O gráfico 3 ilustra a visualização gráfica dos campos de intervenção.

Gráfico 3 - Campos de intervenção

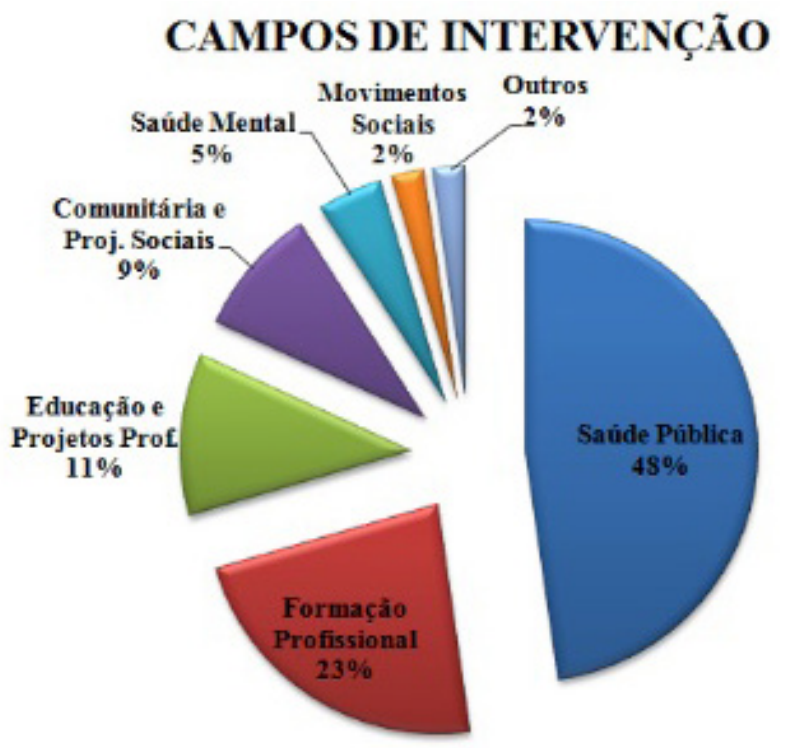


Os artigos que relatavam intervenções foram realizados em diversas instituições como nas unidades da atenção básica de saúde, em alguns centros especializados de saúde, escolas, universidades, secretaria de saúde, unidade de saúde militar, associações, fundações, cooperativas, time de fu- tebol, unidades da assistência social e residência terapêutica.

As instituições foram divididas segundo sua natureza, classificadas como públicas em $70 \%$, privadas em $9 \%$ e cooperativas e organizações sem fins lucrativos em 21\% (gráfico 4).

Gráfico 4 - Natureza das instituições

\section{NATUREZA DAS INSTITUIÇÕES}

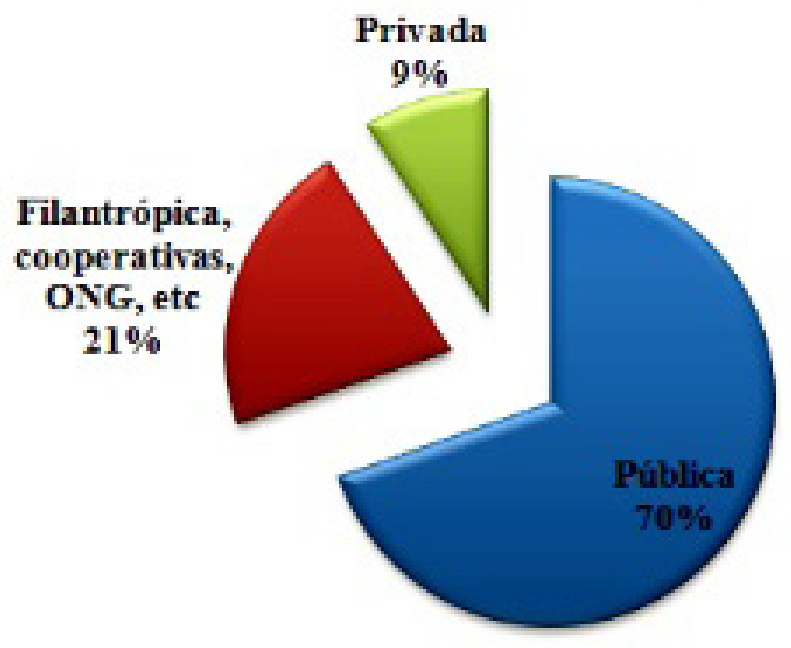

Os públicos-alvo das intervenções foram os seguintes: "pessoas com alguma patologia" (29,04\%); "pessoas em vulnerabilidade social" com (29,04\%); "profissionais da saúde" (19,35\%); "estudantes" (16,12\%); e "outros" $(6,45 \%)$.

Os estados em que ocorreram as intervenções foram Bahia, Ceará, Goiás, Minas Gerais, Paraná, Pernambuco, Rio de Janeiro, Rio Grande do Sul e São Paulo, tendo este último originado $51 \%$ das intervenções publicadas (gráfico 5). 15 ocorreram em capitais de estados, 16 no interior e seis não identificavam o município, sendo São Paulo o estado que mais teve publicações de intervenções em municípios do interior (13). Ribeirão Preto (SP) foi o município em que ocorreram $37,5 \%$ do total das intervenções interioranas publicadas, o maior percentual. Não foram encontrados trabalhos sobre intervenções na região norte do Brasil e apenas dois foram realizados na região centro-oeste do país. 


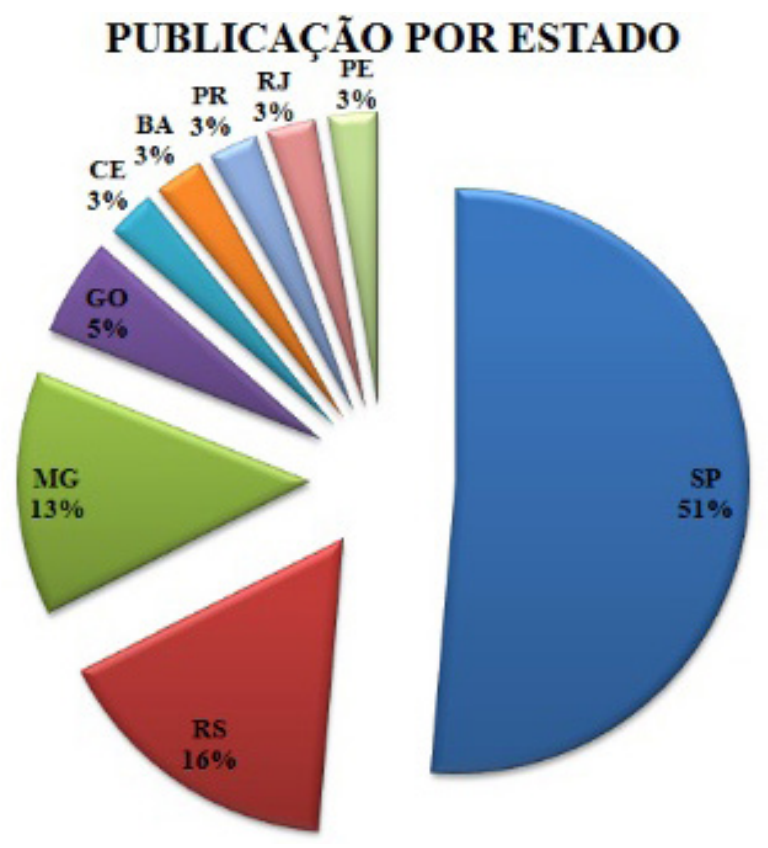

\section{DISCUSSÃO}

A predominância de mulheres autoras dos artigos pesquisados é um fato que pode estar relacionado ao aumento de mulheres pesquisadoras, abordado em estudo de Melo e Oliveira. ${ }^{(9)}$ Além disso, uma vez que a maioria dos artigos foi escrita por profissionais de psicologia e enfermagem, não se deve olvidar a questão do estereótipo das profissões da saúde ou do cuidado serem associadas às mulheres. ${ }^{(9)}$

Essa superioridade do número de publicações por profissionais de psicologia, certamente, foi influenciada pelo acesso ao Pepsic, que é um portal específico desta área. $O$ número também acentuado de profissionais de enfermagem utilizando a psicologia social pichoniana mostra que este não é um recurso apenas para psicólogos, mas uma teoria e técnica de uma escola que "está aberta a todos aqueles que, sejam quais forem seus estudos e formação prévia, se interessem em realizar uma aprendizagem centrada na compreensão dos fenômenos de interação e na análise do processo social $[\ldots] "{ }^{(6)}$

Ainda que o total de profissões dos autores fosse diversificado, o resultado obtido foi de uma pu- jança da saúde sobre as demais áreas do saber, representadas apenas por administração e educação, sinalizando a necessidade de difusão da teoria pichoniana para outros profissionais e/ou da publicação de trabalhos por estes profissionais. Contudo, é preciso pontuar que a veiculação da técnica dos grupos operativos pelas publicações oficiais do Ministério da Saúde pode ter exercido um papel de incentivo aos profissionais da área para trabaIhar com esse dispositivo grupal.

Tratando de formação profissional, deve-se refletir sobre a realização de cursos sobre psicologia social pichoniana pelos profissionais que produzem artigos científicos a esse respeito. Uma vez que essa informação só constava em pequena parcela dos currículos dos autores, pode-se questionar se eles não a incluíram nos seus currículos ou se realmente não possuem formação específica.

Outrossim, ponderando que a técnica de grupos operativos é constituída por um arcabouço teórico complexo, esse resultado pode ilustrar o imperativo de uma formação qualificada por parte dos que utilizam a abordagem pichoniana. Isso porque o reconhecimento da técnica pode não estar sendo acompanhada pelo reconhecimento da capacitação para utilizá-la adequadamente. ${ }^{(7)}$ 
Quanto aos anos em que os artigos foram publicados, percebe-se um crescimento ao passar do tempo, principalmente uma considerável ampliação no século XXI, tendo o ápice das publicações em 2012. Essa multiplicação do número de produções não pressupõe eventos particulares da psicologia social pichoniana, pois acompanha a tendência das publicações no cenário científico brasileiro. (10)

Outro resultado que não é restrito ao objeto de estudo desta pesquisa é o fato de a maioria dos locais de intervenção dos artigos se situar nas regiões sudeste e sul do Brasil, tendo o estado de São Paulo como o que mais reuniu intervenções. Este dado pode não ser restrito à psicologia social pichoniana, mas ilustrar uma situação comum no meio científico brasileiro, pois numa região onde há a maior concentração nacional de cursos de pós-graduação, grupos de estudos e pesquisas e periódicos, ${ }^{(10,11)}$ pode também haver uma maior quantidade de publicações das intervenções pichonianas.

O que não quer dizer que em outras regiões brasileiras não haja a aplicação prática dessa teoria, apenas que não estão sendo publicadas. E essa situação pode estar relacionada ao que Cruz e Stralen (11) argumentam: "A concentração de trabalhos em determinadas regiões e estados [...] é indicativa de um padrão de organização da comunidade científica no Brasil que reflete, em grande medida, diferenças, em particular, das condições socioeconômicas dos estados [...]".

Essa é uma pauta a ser mais bem explorada em estudos específicos sobre possíveis entraves à produção científica em outras regiões do país. No entanto, há espaços de publicações alternativos aos do meio científico, a exemplo de sites de centros de formação. Porém, a divulgação nos meios científicos se mostra relevante para todas as práticas profissionais e se apresenta como uma necessidade também entre os psicólogos sociais. Nascimento ${ }^{(2)}$ aposta na divulgação científica como uma proposta de abertura de diálogo com o interlocutor e afirma que "tem sido pouco frequente entre nós, psicólogos sociais, as tentativas de divulgar, de uma maneira mais sistemática para um público mais amplo, a nossa produção".

Ainda quanto à localidade, cabe abordar que os trabalhos foram realizados de maneira equilibrada entre capitais estaduais e cidades do interior, tendo as últimas superado as primeiras, em quantidade, em alguns estados. Isso evidencia uma distribuição do alcance da psicologia social pichoniana também fora dos grandes centros.

Entre os tipos de estudo, nem todos os artigos utilizaram a técnica dos grupos operativos, o que ratifica que as possibilidades da teoria pichoniana são amplas e vão além da sua técnica. Segundo Ana Quiroga, ${ }^{(13)}$ a psicologia social para Pichon-Rivière não é apenas uma técnica grupal, mas uma reflexão sobre os sujeitos e os seus comportamentos, sendo as suas subjetividades determinadas histórica e socialmente, com o grupo como o horizonte de sua experiência.

Constatou-se que, entre os campos onde ocorreram as intervenções, "saúde pública" e "formação de ensino superior" preponderaram sobre os demais. Não obstante, é pertinente expor que parte significativa das intervenções ocorridas no campo "formação de ensino superior" ocorreu tendo como público-alvo graduandos da área da saúde. Esses dados suscitam a escassez de publicações sobre intervenções em contextos mais variados.

Observando a relação de instituições onde ocorreram as intervenções, nota-se uma diversidade de estabelecimentos especificamente dentro do campo da saúde pública, passando pelos vários níveis de atenção à saúde, desde unidades básicas a hospitais de alta complexidade. No entanto, chama a atenção que não tenha sido encontrado artigo sobre intervenção em CAPS, unidade na qual o Ministério da Saúde prevê a utilização da técnica de grupos operativos, o que aponta uma lacuna para fundamentação da prática neste contexto da saúde mental.

A natureza das instituições em que ocorreram as intervenções, em sua maioria, foi pública e, nos $9 \%$ de artigos sobre intervenções em instituições privadas, os estabelecimentos eram escolas, uni- 
versidades e hospitais particulares. Nenhuma intervenção foi feita em empresa privada. Este é um dado que se relaciona às questões ideológicas da abordagem, uma vez que o trabalho pode ser dificultado se tiver que atender aos interesses da empresa e não dos sujeitos, pois o âmbito empresarial pode impedir que o psicólogo social consiga realizar a promoção de protagonismos dos sujeitos de acordo com o exercício de sua criticidade em relação aos aspectos instrumentais das classes dominantes. ${ }^{(13)}$

O público-alvo que teve mais intervenções foi de pessoas com alguma patologia. Este é um aspecto importante a se refletir, pois apesar de se aprofundar nas particularidades destes públicos, pode-se em algumas situações se perder em termos de heterogeneidade do grupo. E Pichon-Rivière considera que, quanto maior a heterogeneidade, mais homogênea é a tarefa e a produtividade grupal. ${ }^{(6)}$

Além disso, há discussões sobre a relevância de se desenvolver trabalhos que não focalizem as pessoas a partir das suas patologias. Como Witter ${ }^{(14)}$ comenta, "Embora a produção no exterior seja rica e enfoque tanto saúde como doença, no Brasil ela ainda é tímida e tende a olhar mais para a doença". Portanto, para desenvolver a forma de produção brasileira na promoção de saúde, é imprescindível não focalizar os grupos em sua homogeneidade a partir da doença e, sim, a partir de seus aspectos saudáveis.

\section{CONCLUSÃO}

O conjunto dos resultados e suas discussões demonstrou que quase a totalidade dos artigos foram publicados em revistas da área da saúde, e que a autoria está concentrada entre mulheres profissionais da saúde, que realizaram intervenções em instituições públicas, nos campos da saúde e da educação, nas regiões sudeste e sul do Brasil.
Apesar do crescimento no número de publicação sobre essa temática nos últimos anos, os resultados fizeram emergir que, na verdade, carecem publicações sobre intervenções em outros campos e espaços, como comunidades, projetos e movimentos sociais. Também surpreendeu a escassez de trabalhos ligados à saúde mental, principalmente no CAPS, cujo manual do Ministério da Saúde cita a técnica pichoniana.

Faltam dados sobre se parte dos autores possuem formação específica na área ou não, mas, para garantir uma atuação alinhada à psicologia social pichoniana ou mesmo a utilização fidedigna da técnica de grupos operativos, uma capacitação apropriada não pode ser negligenciada, independentemente de ser graduado em área correlata.

Ainda que as práticas tenham sido igualmente numerosas nas capitais e em cidades do interior dos estados, outro ponto com lacunas é a distribuição geográfica não só dentro dos estados, mas também pelas regiões do país. A extensão continental do Brasil comporta culturas múltiplas que, muitas vezes, em determinadas regiões, se atrelam a questões humanas e das formações grupais, merecendo ser contemplada em estudos científicos.

A psicologia social pichoniana não se esgota em ser uma teoria ou técnica dos grupos e nem um complemento da psicologia individual. Ela apresenta conceitos relevantes para se pensar a subjetividade, bem como para exercer uma crítica sobre os fenômenos cotidianos, abordando a dialética entre o intrassubjetivo e o intersubjetivo. (13)

Como foi possível perceber nesta pesquisa, as publicações que utilizam como fundamento a teoria e/ou a técnica de Enrique Pichon-Rivière vem aumentando ao longo do século XXI, o que faz do perfil delineado uma fonte para identificar elementos que demandam uma crítica mais aprofundada sobre os caminhos científicos da psicologia social pichoniana até 2014 e sobre as trajetórias possíveis e necessárias adiante. 


\section{REFERÊNCIAS}

1. Klappebach H. A formação de psicólogos na argentina: a psicologia social de Pichon-Rivière. In: Jacó-Vilela AM, Sato L, organizadores.

Diálogos em psicologia social. Rio de Janeiro: Centro Edelstein de Pesquisas Sociais; 2012. p. 168-200.

2. Ministério da Saúde (BR). Secretaria de Atenção à Saúde. O humanizaSUS na atenção básica. Brasília, DF: Ministério da Saúde; 2009. (Série B. Textos básicos de saúde; 1)

3. Ministério da Saúde (BR). Secretaria de Atenção à Saúde. O humanizaSUS na atenção básica. Brasília, DF: Ministério da Saúde; 2010. (Série B. Textos básicos de saúde; 2).

4. Ministério da Saúde (BR). Secretaria de Atenção à Saúde. Departamento de Ações Programáticas Estratégicas. Saúde mental no SUS: os centros de atenção psicossocial. Brasília, DF: Ministério da Saúde; 2004. (Série F. Comunicação e educação em saúde).

5. Ministério da Saúde (BR). Portaria $n^{\circ} 121$, de 25 de janeiro de 2012. Institui a unidade de acolhimento para pessoas com necessidades decorrentes do uso de crack, álcool e outras drogas, no componente e atenção residencial de caráter transitório da Rede de Atenção Psicossocial. Brasília, DF: Ministério da Saúde; 2012.

6. Pichon-Rivière E. O processo grupal. Velloso MAF, tradutor. São Paulo: Martins Fontes; 1980/1988.

7. Chatelain LG. Psicología social y grupo operativo: una disociación inoperante. In: Cortés J, coordenador. Congreso Internacional de Grupos Operativos: Intervención Grupal y Poder. Santiago, Chile: 2014.
8. Gil AC. Como elaborar projetos de pesquisa. $4^{\mathrm{a}}$. ed. São Paulo: Atlas; 2002.

9. Melo HP, Oliveira AB. A produção científica brasileira no feminino [Internet]. Cad.

Pagu; 2006; 27:301-331. Disponível em: http://www.scielo.br/scielo.php? script=sci_ arttext\&pid=SO104-83332006000200012\&ln $\mathrm{g}=\mathrm{pt \& nrm}=$ iso\&tlng=pt

1O. Bezerra MLS, Neves EB. Perfil da produção científica em saúde do trabalhador [Internet]. Saúde Soc; 2O1O;19(2):384-394. Disponível em: http://www.scielo.br/scielo.php? script=sci_ arttext\&pid=SO1O4-129O2O1000O2OOO14\&ln $\mathrm{g}=\mathrm{pt \& nrm}=$ iso\&tlng=pt

11. Cruz RN, Stralen CJV. A produção do conhecimento na psicologia social brasileira: um estudo descritivo a partir da revista psicologia \& sociedade, 1986-1992 [Internet]. Psicol. soc. 2O12; 24(1):227-239. Disponível em: http://www.scielo.br/ scielo.php?script=sci_ arttext\&pid=SO1O2-71822O12000100O25\&lng =pt\&nrm=iso\&tl ng=en

12. Nascimento ARA. Psicologia social e divulgação científica: rupturas e pontos de contato. In: Medrado R, Galindo W, organizadores. Psicologia social e seus movimentos: 30 anos de ABRAPSO. Pernambuco: Universitária; 2011. p. $111-120$.

13. Quiroga APD. Enfoques y perspectivas en psicología social: desarrollos a partir del pensamiento de Enrique Pichon-Rivière. Buenos Aires: Cinco; 2009.

14. Witter GP. Psicologia da saúde e produção científica [Internet]. Estud. psicol. (Campinas); 2008; 25(4):577-584. Disponível em: http://www.scielo.br/scielo.php? script=sci_ arttext\&pid=S0103-166X2008000400012\&ln $\mathrm{g}=\mathrm{pt \& nrm}=$ iso\&tlng=pt 